\title{
Look out: An exploratory study assessing how gaze (eye angle and head angle) and gait speed are influenced by surface complexity
}

\author{
Nicholas Thomas ${ }^{\text {Corresp., }, 2}$, James Gardiner $^{2}$, Robin Crompton ${ }^{2}$, Rebecca Lawson ${ }^{1}$ \\ 1 Institute of Population Health Sciences, University of Liverpool, Liverpool, United Kingdom \\ 2 Institute of Ageing \& Chronic Disease, University of Liverpool, Liverpool, United Kingdom \\ Corresponding Author: Nicholas Thomas \\ Email address: N.Thomas3@liverpool.ac.uk
}

Background: Most research investigating the connection between walking and visual behaviour has assessed only eye movements (not head orientation) in respect to locomotion over smooth surfaces in a laboratory. This is unlikely to reflect gaze changes found over the complex surfaces experienced in the real world, especially given that eye and head movements have rarely been assessed simultaneously.

Research question: How does gaze (eye and head) angle and gait speed change when walking over surfaces of different complexity?

Methods: In this exploratory study, we used a mobile eye tracker to monitor eye movements and inertial motion sensors (IMUs) to measure head angle whilst subjects $(n=11)$ walked over surfaces with different complexities both indoors and outdoors. Gait speed was recorded from ankle IMUs.

Results: Overall, mean gaze angle was lowest over the most complex surface and this surface also elicited the slowest mean gait speed. The head contributed increasingly to the lowering of gaze with increased surface complexity. Less complex surfaces showed no significant difference between gaze and gait behaviour.

Significance: This study supports previous research showing that increased surface complexity is an important factor in determining gaze and gait behaviour. Moreover, it provides the novel finding that head movements provide important contributions to gaze location. Our future research aims are to further assess the role of the head in determining gaze location during locomotion across a greater range of complex surfaces to determine the key surface characteristics that influence gaze during gait. 


\section{Look out: An exploratory study assessing how gaze (eye}

\section{2 angle and head angle) and gait speed are influenced by}

\section{3 surface complexity}

\section{4}

5 Nicholas D. A. Thomas ${ }^{1,2 *}$, James, D. Gardiner ${ }^{2}$, Robin H. Crompton ${ }^{2}$, \& Rebecca Lawson ${ }^{1}$

$7 \quad{ }^{1}$ Institute of Population Health Sciences, University of Liverpool, Liverpool, UK

$8 \quad{ }^{2}$ Institute of Ageing \& Chronic Disease, University of Liverpool, Liverpool, UK

Corresponding Author:

12 William Henry Duncan Building, Institute of Ageing \& Chronic Disease, University of 
25

26

27

\section{Abstract}

Background: Most research investigating the connection between walking and visual behaviour has assessed only eye movements (not head orientation) in respect to locomotion over smooth surfaces in a laboratory. This is unlikely to reflect gaze changes found over the complex surfaces experienced in the real world, especially given that eye and head movements have rarely been assessed simultaneously.

Research question: How does gaze (eye and head) angle and gait speed change when walking over surfaces of different complexity?

Methods: In this exploratory study, we used a mobile eye tracker to monitor eye movements and inertial motion sensors (IMUs) to measure head angle whilst subjects $(n=11)$ walked over surfaces with different complexities both indoors and outdoors. Gait speed was recorded from ankle IMUs.

Results: Overall, mean gaze angle was lowest over the most complex surface and this surface also elicited the slowest mean gait speed. The head contributed increasingly to the lowering of gaze with increased surface complexity. Less complex surfaces showed no significant difference between gaze and gait behaviour.

Significance: This study supports previous research showing that increased surface complexity is an important factor in determining gaze and gait behaviour. Moreover, it provides the novel finding that head movements provide important contributions to gaze location. Our future research aims are to further assess the role of the head in determining gaze location during locomotion across a greater range of complex surfaces to determine the key surface characteristics that influence gaze during gait. 


\section{Introduction}

53

54

Our ability to understand how people walk through their environment should be informed not only by assessment of their gait but by understanding the visual information available to them. Visual information is particularly important when environments are more complex, requiring increased planning to maintain stability whilst walking. For example, spatial and temporal visual information has been shown to be essential for correct foot positioning over complex surfaces, both inside and outside of the laboratory [1-3]. Complex surfaces increase fall risk for all age groups as a result of poor stability $[4,5]$. Therefore, an increased understanding of how vision and gait are impacted by different surfaces is important to help to understand and prevent falls.

Research investigating gait and gaze often uses terminology inconsistently. Here, we will use 'complex' to refer to all non-smooth surfaces. These include surfaces with slope changes [6], inconsistently spaced foot targets $[1,7]$, uneven surfaces [8] and combinations of these features $[9,10]$. Smooth surfaces here are taken to include even, horizontal surfaces in laboratories [9], on walkways [11] and outside [12]. Lastly, while gaze is often used by researchers to refer only to eye movements, here we define gaze as the orientation of the eye in a world reference frame. Gaze thus combines eye-in-head movements and head-in-world movements, which we measured using an eye tracker and an inertia measurement unit sensor (IMU) respectively.

Most gait research uses a smooth, horizontal, hard laboratory floor. However, some laboratory-based studies have started to address how conditions more representative of realworld surfaces may impact our behaviour. These studies have not, though, produced consistent findings. For example, Menant, Steele [13] found that gait speed decreased over complex 
74 surfaces, but this finding was not supported by the work of Thies, Richardson [8]. These

75

76

77

78

79

80

81

82

differences may have arisen because there are no standards for defining complex surfaces in terms of roughness, slope, etc. In contrast to studies of gait, studies investigating gaze during walking have shown a clearer consensus. Compared to smooth surfaces, complex surfaces have been shown to cause eye movements to be increasingly directed to the ground, to lead to increased numbers of fixations, and to require visual information from at least two steps ahead for safe and efficient locomotion $[1,3,9,14]$.

Crucially, it is not known whether laboratory simulations accurately represent the surfaces over which we typically walk in everyday life. An alternative, and more ecologically valid, approach to using mixed surface conditions inside the laboratory is to conduct experiments outside. 't Hart and Einhauser [15] assessed gaze for individuals walking outdoors on irregularly placed steps and a smooth road. They reported that their complex surfaces caused individuals to lower both their eyes and head. The eyes lowered more than the head, suggesting that the eyes served more immediate demands when walking. Note, though, that this study only indirectly measured head movements by inferring them from the output of the scene view camera attached to the eye-tracker. Thus, we do not yet have an accurate understanding of how the head affects overall gaze.

Although the results of 't Hart and Einhauser [15] suggest that the head plays an important role in altering gaze when traversing complex surfaces, few other studies have investigated the importance of head movements, independent of eye movements, in contributing to overall gaze. For example, Matthis and Fajen [1] \& Marigold and Patla [9] only considered eye movements during walking over complex surfaces. Other studies have inferred head movement from movements of the world camera attached to the eye tracker $[15,16]$. In the 
97 present study we follow Matthis, Yates [3] using an alternative approach that allowed us to

98 measure head movements independent of the eye tracker whilst walking over complex surfaces.

99 This methodology to calculate gaze has been previously used for tasks other than walking over

100 complex surfaces, for example see $[17,18]$. Head movements are particularly important to

101 consider given that weakened musculoskeletal health, including age associated declines, might

102 limit head movement, and this, in turn, could impact gaze. Tomasi, Pundlik [19] assessed head

103 movements, using IMUs, whilst also tracking eye movements. They found that over $40 \%$ of gaze

104 movement was due to head movements when walking outdoors. This study did not, though,

105 measure other behaviour changes which are also likely to be important to understanding the

106 relation between locomotion and gaze behaviour, such as speed of locomotion and changes in

107 stride length or timing. Moreover, Tomasi, Pundlik [19] only analysed head yaw (left to right,

108 horizontal movement), whereas head pitch (up and down, vertical movement) is likely to be

109 more important when traversing non-smooth surfaces [15]. Common sense would dictate that

110 movement of the eyes to change vertical gaze orientation are more energy efficient than

111 movement of the head, which requires the activation of more and larger, muscles for the same

112 effect on gaze location. However, to the authors' knowledge, no study has accurately assessed

113 eye angle and head angle when walking over complex surfaces. Thus, it remains unclear how eye

114 and head angle contribute to gaze when walking over different surfaces.

On the basis of the above we believe it is important to independently assess head as well

116 as eye movements to understand how surface complexity influences gaze, and to see how this

117 relates to changes in gait. As an initial step, in this exploratory study, people walked in a straight

118 line on four horizontal surfaces at self-paced speeds. We measured changes in vertical eye angle

119 and head pitch angle, as well as the gait speed of participants. Here, we focus on presenting 
120 results for mean values across a trial walk for eye angles, head pitch angles, gaze (combined eye

121 and head pitch) angles and gait speed, as our aim was to compare overall performances across

122 different surface complexities. In future work we aim to conduct more fine-grained analyses of

123 short term, step by step changes in the relation between eye and head pitch angles and gait. For

124 eye and head angle, only vertical change was assessed as horizontal movements are unlikely to

125 be associated with maintaining stability during straight line walking. Thus, in summary, we

126 assessed how eye and head movements independently contribute to gaze, and how this relates to

127 changes in gait speed during locomotion over surfaces of different complexity.

\section{Methodology}

130

131

132

133

134

135

136

137

138

139

140

141

\section{Participants}

11 healthy adults $(7$ male, mean $\pm \mathrm{SD}$; age $=24.6 \pm 3.5$ years; height $=173 \pm 6.5 \mathrm{~cm})$ were recruited for this exploratory study. Data from 9 more participants was collected but was not used due to a malfunction of the inertia sensors (both the gyroscopic and accelerometric data recorded for these participants produced extreme values, far exceeding the normal range in all trials). For ease of recording data with the eye tracker, only participants who did not require glasses for everyday walking were selected. No participant had an injury or impairment that affected their gait or vision.

\section{Data Collection}

Ethical approval for the study was obtained from the University of Liverpool's Ethics Committee (REF: 1900). Two IMU sensors (Delsys TRIGNO ${ }^{\mathrm{TM}}$ IM, Boston, MA, USA) were 
142 positioned on the participant. Each sensor consisted of a 3-axis accelerometer, gyroscope and

143 magnetometer, recording at $148 \mathrm{~Hz}$. One IMU was positioned close to the midline of the forehead

144 to calculate head pitch angle using gyroscopic data. The second IMU was positioned above the

145 lateral malleoli on the left shank and was used to calculate gait events. Participants wore an

146 Arrington Research ViewPoint (Scottsdale, Arizona, USA) eye tracker that recorded pupil

147 movement at $60 \mathrm{~Hz}$ and a scene camera that recorded the participant's view of the environment

148 that recorded at $30 \mathrm{~Hz}$. Eye angles in the vertical direction were calculated in order to calculate

149 how far ahead on the ground participants were looking as they walked.

150 Protocol

151 The eye-tracker was calibrated prior to each data collection session. Eye movements were

152 calculated based on the dimensions on the screen used in the calibration, see supplementary

153 material S1. Participants then walked ten times over four different surfaces so they each

154 completed 40 trials in total. The surfaces comprised an uneven, indoor, and a flat, indoor surface,

155 both in a gait laboratory, and then a paved, outdoor, and a cobbled, outdoor surface, both on the

156 university campus (Figure 1a-d). The indoor, flat surface (13.20m long) consisted of eleven

$15718 \mathrm{~mm}$ thick medium density fibreboards (MDF) panels. The indoor, uneven surface was

158 identical except that each panel had an array of blocks of 9mm thick MDF on top of the base

159 layer to give an uneven surface with a maximum height range of $27 \mathrm{~mm}$. Each panel had the same

160 block design, with blocks spaced to prevent participants from easily targeting footfalls whilst

161 walking. The outdoor, paved surface $(16.60 \mathrm{~m}$ long) comprised paving stones $(60 \times 60 \mathrm{~cm})$ whilst

162 the outdoor, cobbled surface (15.70m long) comprised of setts. All surfaces were long enough to

163 ensure participants could achieve a steady state of walking [20]. 
placed at either the start or end of each surface. This obstacle was intended to increase surface

end) did not show a strong or clear relationship with either gaze angle or speed across any of the

four surfaces so this manipulation was not included in the analysis presented here.

comfortable speed along the surface before looking straight ahead whilst standing still at the end

of the surface for three seconds. No instructions were given regarding head or eye movement when walking.

Data Analysis

Mean eye angle and head pitch angle were calculated for each trial of each surface for all participants. For the raw vertical gyroscopic data used to determine head pitch angle, a low pass, $10 \mathrm{~Hz}$ fourth-order Butterworth filter was used to reduce noise. The effect of drift was removed using gyroscopic data taken from the period when the participant remained still at the start and end of each trial (following Takeda, Lisco [21]). The gyroscopic data (in degrees per second) was then numerically integrated over the trial to give head pitch angle. The supplementary material (S2) describes a check of the accuracy of this method. The vertical eye movements were converted into angular data. A head pitch angle of $0^{\circ}$ was defined as the average head orientation at the beginning and end of each trial when the participant remained still whilst looking straight ahead. To avoid the influence of starting and stopping, the walking data was trimmed to remove the first two and last two strides from each trial. Every 1/60s during each trial the eye angle and 
187 head angle were summed and these sums were then averaged across the trial to calculate gaze 188 (combined eye and head pitch) angle for that trial. The relative frequency distribution of eye, 189 head pitch and gaze angles for each surface were also calculated. This measurement follows 190 Foulsham, Walker [22] in calculating the frequency of recorded angles for each surface in bins of $1915^{\circ}$ relative to zero. In effect this distribution shows the variance of eye and head movement 192 during the trial. Only eye angles that were within the normal range expected based on previous 193 reports [23] and from our own validation study, see supplementary material (S3), were included. combining this with integrated accelerometery data (following Li, Young [24]). As this method has only been tested over smooth surfaces, we checked its accuracy over the most complex surface, the indoor, uneven surface, as detailed in the supplementary material (S2). pitch angles and gaze angles. The factor of surface had four levels: flat, paved, cobbled and uneven. Correlations were calculated between eye angle and head pitch angle every $1 / 60$ s of the trial for all participants. We then conducted t-tests for each surface to compare the mean correlation across participants to a no correlation value (zero). Zero correlation would suggest that there was no relation between eye angle and head pitch angle for that surface. A repeatedmeasures ANOVA was conducted for the participant's mean gait speed with a factor of surface. Finally, correlations were calculated between mean speed and mean eye angle, mean head pitch angle, and mean gaze, followed by t-tests for each surface to compare the mean correlation across participants to a no correlation value (zero). These correlations were calculated between mean values over a whole trial since speed was calculated across step duration whereas eye, head pitch and gaze angles were calculated every $1 / 60 \mathrm{~s}$. 


\section{Results}

211 Analysis of the orientation of eye, head pitch and gaze (combined eye and head pitch) angles

212

Comparisons were made between all four surfaces. Mean $( \pm \mathrm{SE})$ eye $(\alpha / \mathrm{red})$, head pitch

$214\left(\theta\right.$ /blue) and gaze (grey) angles $\left(^{\circ}\right)$ are shown in Figure 2. Surface had a significant effect on

215 gaze angle, $\mathrm{F}(3,30)=28.34, \eta_{\mathrm{p}}{ }^{2}=0.81, p=0.003$. Post-hoc Newman Keuls tests $(\mathrm{p}<0.05)$

216 showed gaze to be significantly lower for the indoor, uneven surface compared to the other three

217 surfaces. The contribution to mean gaze angle from head pitch $(\theta)$ angle changes were $17 \%$ for

218 indoor, flat surfaces; $25 \%$ for outdoor, paved surfaces; $35 \%$ for outdoor, cobbled surfaces; and

$21954 \%$ for indoor, uneven surfaces. This contribution was calculated as the percentage of the head

220 pitch angle compared to the gaze (combined eye and head pitch) angle taken every 1/60s over the

221 course of every trial and then averaged. The average frequency distribution of eye, head pitch

222 and gaze angles over the trial was calculated for each surface, see Figure 3. The indoor, uneven

223 surface had a different distribution to the other three surfaces. These other surfaces all had peak

224 head pitch angles close to zero, whereas the indoor uneven surface showed a greater range of

225 head pitch angles. For this surface, head pitch angle was often lowered, with a similar range

226 distribution to that for eye angle. Gaze (combined eye and head pitch) angle showed a similar

227 distribution to eye angle for all but the indoor, uneven surface where it was generally lower.

A one sample t test showed that the correlation between eye and head pitch angle for the

229 indoor, flat $(\mathrm{M} \pm \mathrm{SD}=+0.13 \pm 0.17)$, and outdoor, paved surface, $(+0.24 \pm 0.15)$ were

230 significantly greater than zero $(\mathrm{t}(10)=2.46, p=0.034$ and $\mathrm{t}(10)=5.63, p<0.01$ respectively).

231 These correlations, albeit weak, suggest that eye and head movements are co-ordinated when

232 walking over these surfaces. The correlations for the indoor, uneven $(+0.01 \pm 0.17 ; \mathrm{t}(10)=0.26$, 
$233 p=0.801)$ and outdoor, cobbled $(+0.15 \pm 0.23 ; \mathrm{t}(10)=2.12, p=0.060)$ surfaces were not

234 significantly different to zero. As all four correlations were all relatively low, this suggests that 235 eye angle and head pitch angle both contribute distinct information about gaze angle.

237 Gait speed analyses

Speeds were significantly different across surfaces, $\mathrm{F}(3,30)=38.40, \eta_{\mathrm{p}}{ }^{2}=0.89 p<0.001$, as shown in Figure. 4. A post-hoc Newman Keuls test $(\mathrm{p}<0.05)$ showed participants walked more slowly on the indoor, uneven surface $(\mathrm{M} \pm \mathrm{SE}=1.19$ metres/second \pm 0.05$)$ than the indoor, flat $(1.35 \pm 0.04)$, outdoor, paved $(1.43 \pm 0.04)$ and outdoor, cobbled $(1.44 \pm 0.04)$

242 surfaces. gaze angle over the trial. One sample t-tests revealed that no correlations with speed were significantly different from zero, see Table 1.

We estimated how long it would take participants to walk to the location that they were fixating for each surface. To do this, we used the average participant eye height and their mean gaze (combined eye and head pitch) angle to calculate the mean distance that participants were looking ahead for each surface. We then divided this distance by the average participant gait speed for that surface to estimate how long it would take for participants to walk to their fixation

251 location, see Table 2. This was shortest for the indoor uneven surface. Similarly, using the 252 average step length for each surface, we calculated how many steps people looked ahead. People 253 looked fewer steps ahead on the indoor uneven surfaces, see Table 2. 


\section{Discussion}

255 The aim of this exploratory study was to understand how eye angle and head pitch angle

256 contribute to gaze behaviour and how this alters with gait speed when walking over surfaces of

257 different complexity. When traversing the most complex surface (indoor, uneven; mean height

258 change $=7.46^{\circ}$, see Figure 1), participants significantly lowered their gaze (combined eye and

259 head pitch) angle and reduced their gait speed. Head pitch angle was lowered towards the ground

260 for a greater duration of the trial over this surface (as shown by the relative frequency

261 distribution, see Figure 3), and a greater proportion of gaze angle was attributed to head pitch

262 angle than for any other surface (54\%). Our results suggest that more complex surfaces require

263 greater visual information to traverse, with a stronger contribution to overall gaze angle being

264 made by head pitch angle in such circumstances.

The results in our study are consistent with previous research in showing that complex

surfaces exert increased visual demands [3, 9, 15] as it becomes harder to maintain stability.

267 Using mean values of gaze (combined eye and head pitch) angle and speed, we showed that

268 participants walking over the indoor, uneven surface looked just two steps ahead (see Table 1).

269 This finding is in line with that previously reported when walking on inconsistently spaced foot

270 holds $[1,2]$. Further research is required to test how different characteristics of irregular surfaces

271 (slope, unevenness, appearance, texture, etc.) influence eye and head pitch behaviour. The

272 present study only measured surfaces by changes in their mean height. An important future goal

273 will be to characterise surfaces using comprehensive, objective and replicable measures.

A relatively novel aspect of the current study was analysing eye and head pitch angle

275 independently when walking over different surfaces. Our results found no strong relation

276 between eye and head pitch angle (note, though, that our analyses could not detect short-term 
277 correlations). Only two surfaces (indoor, flat and outdoor, paved) produced a significant

278 correlation between eye and head pitch angles and these correlations were weak. For these

279 simpler surfaces there was some evidence that eye and head movements were co-ordinated. This

280 might reflect participants spending more time gazing around the scene rather than having to

281 fixate near to their upcoming foot placements on these less challenging surfaces. The relative

282 frequency plots (see Figure 3) showed differences between eye and head pitch angles. The eyes

283 were typically lowered more than the head except when walking over the most complex indoor,

284 uneven surface. This suggests that the energetically costly movement of the head to shift gaze is

285 only implemented when necessary, i.e. when surfaces are more complex to traverse,

286

287

288

289

290

291

292

293

294

295

296

297

298

299

compromising stability. This supports findings from 't Hart and Einhauser [15], showing eye

movements are usually greater than head movements. Furthermore, these results strengthens the rationale of Tomasi, Pundlik [19] for calculating gaze from both eye and head movements. The lack of contribution from the head to overall gaze when walking over smooth surfaces may suggest that our peripheral vision is sufficient in these settings. Indeed, peripheral vision has been shown to be sufficient even when traversing an obstacles [11]. Future research is therefore required to determine how complex surfaces must be in order to elicit lowering of the head.

In our study, changes in eye angle, head pitch angle and gait speed were assessed from mean values across the entire length of the surface traversed on a given trial. We found significant differences between surfaces using this approach (see Figure 2), and we believe that this summary measure provides a convenient and meaningful summary of gaze behaviour over different surfaces. Surface lengths changed slightly between surfaces, but given that we excluded data from the start and end of the surface, differences of surface complexity are likely to be the main cause of behavioural change. 
A more detailed approach to determine gaze behaviour can come from time series data,

301 for example as used by Matthis, Yates [3]. The supplementary material (S4) shows an example

302 of time series data from our study, plotting raw gaze angle for ten trials of one participant

303 walking over the outdoor, cobbled and the indoor, uneven surfaces. For this participant, gaze was

304 consistently lower for the indoor, uneven surface compared to the outdoor, paved surface, whilst

305 overall gaze angles were generally lower.

306

307

308

309

310

311

312

313

314

315

316

317

\section{Conclusions}

In summary, we found gaze and gait behaviour to be most affected when participants walked on a complex, uneven surface. In this situation both head and eye movements played a substantial role in determining gaze angle, supporting the argument [19] that we should not assess gaze solely by considering eye movements. This research should act as a foundation for future work to tease apart what surface characteristics drive behavioural changes in gaze and gait when we walk over the types of surfaces that we commonly encounter in our everyday lives (e.g. slopes, cobbles, steps).

\section{Acknowledgements}

The authors wish to thank Jenni Delight and Alexandra Allen for their assistance.

\section{References}

[1] Matthis JS, Fajen BR. Visual Control of Foot Placement When Walking Over Complex Terrain. J Exp Psychol Human. 2014;40:106-15.

[2] Matthis JS, Barton SL, Fajen BR. The critical phase for visual control of human walking over complex terrain. Proc Natl Acad Sci U S A. 2017;114:E6720-E9.

[3] Matthis JS, Yates JL, Hayhoe MM. Gaze and the Control of Foot Placement When Walking in Natural Terrain. Curr Biol. 2018;28:1224-33 e5. 
325

326

327

328

329

330

331

332

333

334

335

336

337

338

339

340

341

342

343

344

345

346

347

348

349

350

351

352

353

354

355

356

357

358

359

360

361

362

363

364

365

366

367

[4] Nyman SR, Ballinger C, Phillips JE, Newton R. Characteristics of outdoor falls among older people: a qualitative study. Bmc Geriatr. 2013;13.

[5] Talbot LA, Musiol RJ, Witham EK, Metter EJ. Falls in young, middle-aged and older community dwelling adults: perceived cause, environmental factors and injury. Bmc Public Health. 2005;5.

[6] Merryweather A, Yoo B, Bloswick D. Gait Characteristics Associated with Trip-Induced Falls on Level and Sloped Irregular Surfaces. Mineral-Basel. 2011;1:109-21.

[7] Patla AE, Vickers JN. How far ahead do we look when required to step on specific locations in the travel path during locomotion? Exp Brain Res. 2003;148:133-8.

[8] Thies SB, Richardson JK, Ashton-Miller JA. Effects of surface irregularity and lighting on step variability during gait: A study in healthy young and older women. Gait Posture. 2005;22:26-31.

[9] Marigold DS, Patla AE. Gaze fixation patterns for negotiating complex ground terrain. Neuroscience. 2007;144:302-13.

[10] Marigold DS, Patla AE. Age-related changes in gait for multi-surface terrain. Gait Posture. 2008;27:689-96.

[11] Graci V, Elliott DB, Buckley JG. Utility of peripheral visual cues in planning and controlling adaptive gait. Optom Vis Sci. 2010;87:21-7.

[12] Storm FA, Buckley CJ, Mazza C. Gait event detection in laboratory and real life settings: Accuracy of ankle and waist sensor based methods. Gait Posture. 2016;50:42-6.

[13] Menant JC, Steele JR, Menz HB, Munro BJ, Lord SR. Effects of walking surfaces and footwear on temporo-spatial gait parameters in young and older people. Gait Posture. 2009;29:392-7.

[14] Matthis JS, Barton SL, Fajen BR. The biomechanics of walking shape the use of visual information during locomotion over complex terrain. J Vision. 2015;15:10-.

[15] 't Hart BM, Einhauser W. Mind the step: complementary effects of an implicit task on eye and head movements in real-life gaze allocation. Exp Brain Res. 2012;223:233-49.

[16] Elloumi W, Treuillet S, Leconge R. Real-Time Estimation of Camera Orientation by Tracking Orthogonal Vanishing Points in Videos. VISAPP (2)2013. p. 215-22.

[17] Fang Y, Nakashima R, Matsumiya K, Kuriki I, Shioiri S. Eye-head coordination for visual cognitive processing. Plos One. 2015;10:e0121035.

[18] Land MF. Predictable eye-head coordination during driving. Nature. 1992;359:318.

[19] Tomasi M, Pundlik S, Bowers AR, Peli E, Luo G. Mobile gaze tracking system for outdoor walking behavioral studies. J Vision. 2016;16.

[20] Najjar M, Iman-Eini H, Moeini A. Increasing the Range of Modulation Indices with the Polarities of Cells and Switching Constraint Reliefs for the Selective Harmonic Elimination Pulse Width Modulation Technique. J Power Electron. 2017;17:933-41.

[21] Takeda R, Lisco G, Fujisawa T, Gastaldi L, Tohyama H, Tadano S. Drift Removal for Improving the Accuracy of Gait Parameters Using Wearable Sensor Systems. Sensors-Basel. 2014;14:23230-47.

[22] Foulsham T, Walker E, Kingstone A. The where, what and when of gaze allocation in the lab and the natural environment. Vision research. 2011;51:1920-31.

[23] Lee WJ, Kim JH, Shin YU, Hwang S, Lim HW. Differences in eye movement range based on age and gaze direction. Eye. 2019:1.

[24] Li $Q$, Young $M$, Naing V, Donelan JM. Walking speed estimation using a shank-mounted inertial measurement unit. J Biomech. 2010;43:1640-3. 


\section{Figure 1}

Figure 1

Images showing the four surfaces: $(A)$ indoor, flat, $(B)$ outdoor, paved $(C)$ outdoor, cobbled and (D) indoor, uneven. To estimate surface roughness, we used a clinometer to take 20 measurements of the height change between a pair of points that were $15 \mathrm{~cm}$ apart. This was done at $30 \mathrm{~cm}$ intervals along each surface. The mean $( \pm S D)$ height change was $1.8^{\circ}\left( \pm 0.5^{\circ}\right)$ for the indoor, flat surface, $1.9^{\circ}\left( \pm 0.5^{\circ}\right)$ for the outdoor, paved surface, $2.5^{\circ}\left( \pm 1.9^{\circ}\right)$ for the outdoor, cobbled surface and $7.5^{\circ}\left( \pm 2.6^{\circ}\right)$ for the indoor, uneven surface.

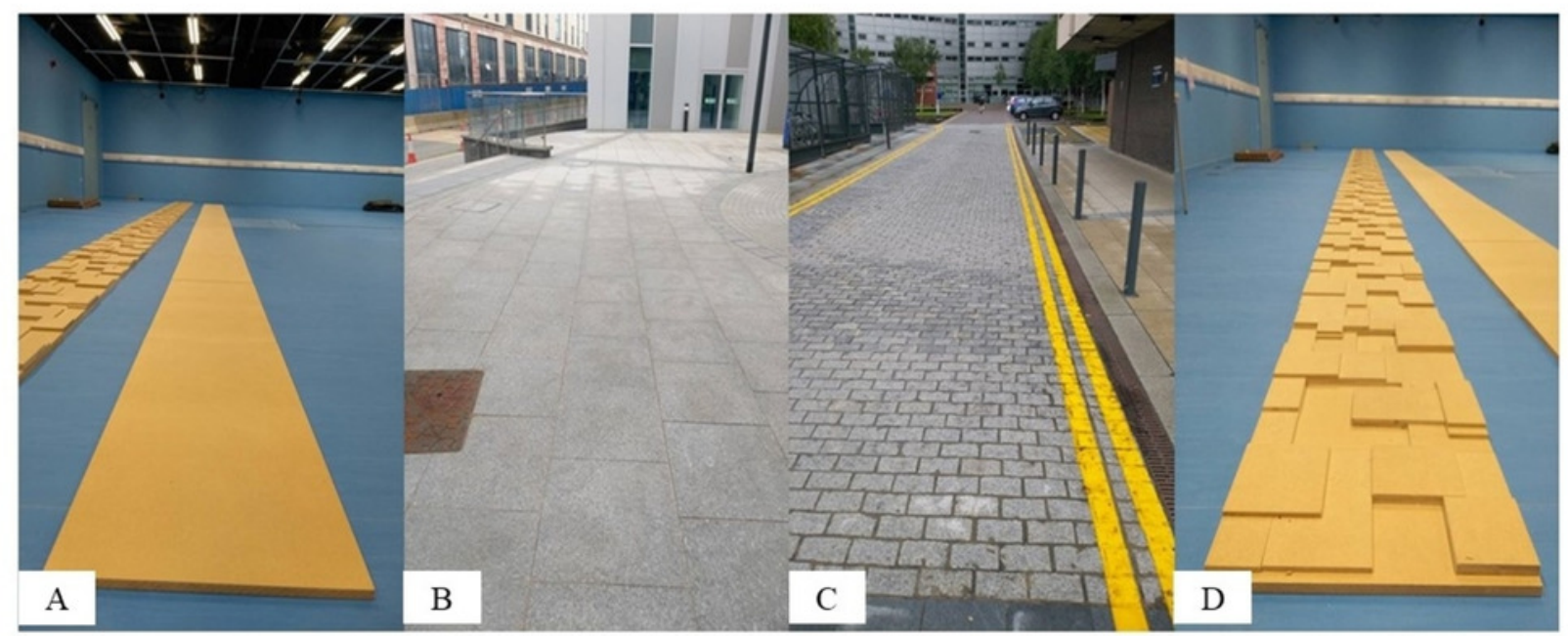




\section{Figure 2}

Figure 2

Mean ( \pm SE) eye, head pitch and gaze (combined eye and head pitch) angles $\left({ }^{\circ}\right.$ ) for the four surfaces tested: indoor, flat; outdoor, paved; outdoor, cobbled; and indoor, uneven. The inset shows how eye $(\alpha /$ red) and head pitch $(\theta /$ blue) angles were measured. Mean gaze (combined eye and head pitch) angle is the mean value of the sum of eye angle and head pitch angle calculated every $1 / 60$ s (and not the sum of the mean eye angle and mean head pitch angle).

Surfaces

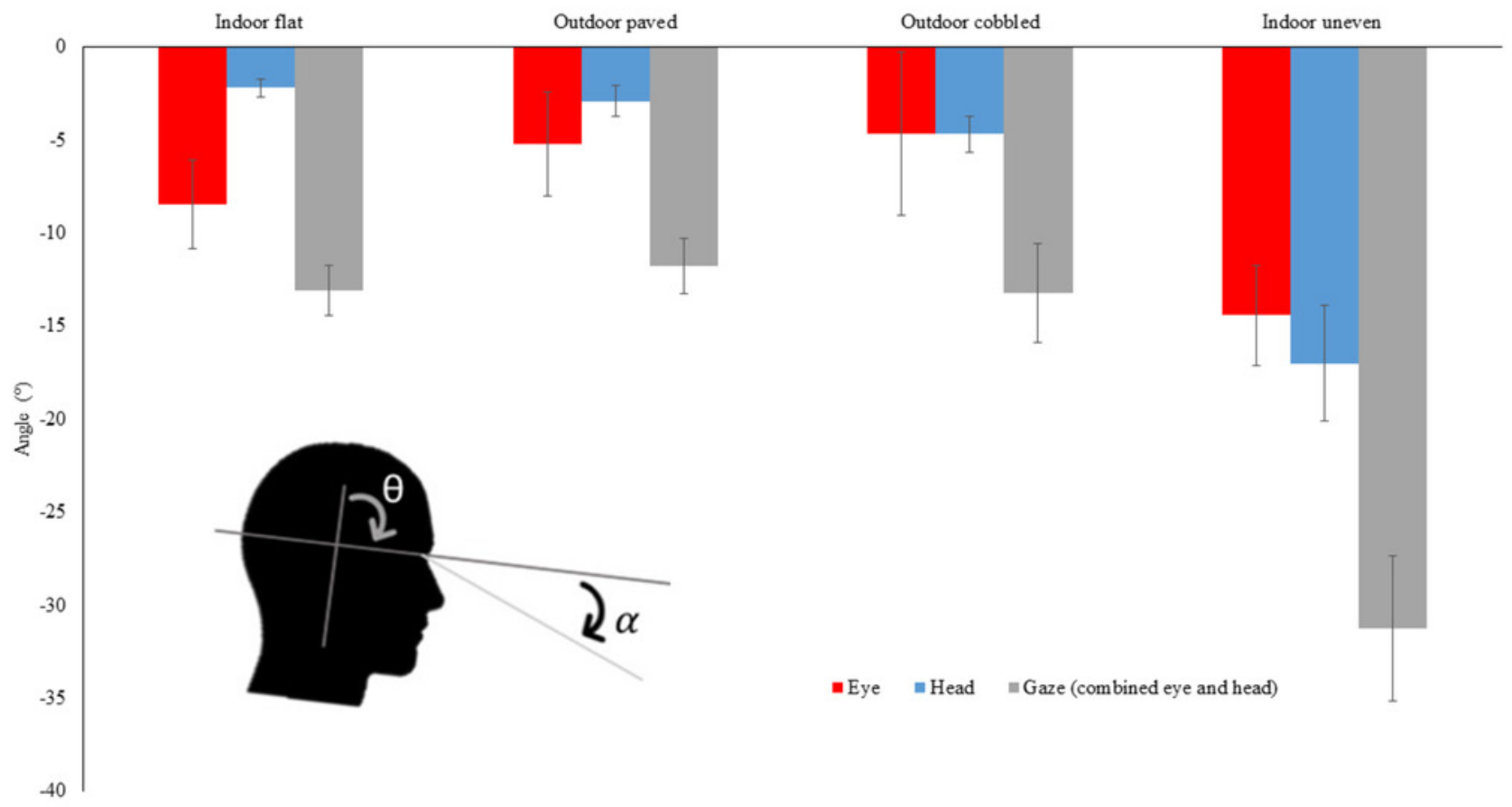




\section{Figure 3}

Figure 3.

Relative frequency distributions of eye, head pitch and gaze (combined eye and head pitch) angles $\left({ }^{\circ}\right)$, within a trial for the (A) indoor, flat, (B) outdoor, paved, (C) outdoor, cobbled and (D) indoor, uneven surfaces. Negative angles correspond to lowering of the eyes and head toward the ground. An angle of zero (indicated by the black dashed line) represents the mean angle as the participant looked ahead at the start and end of each trial.
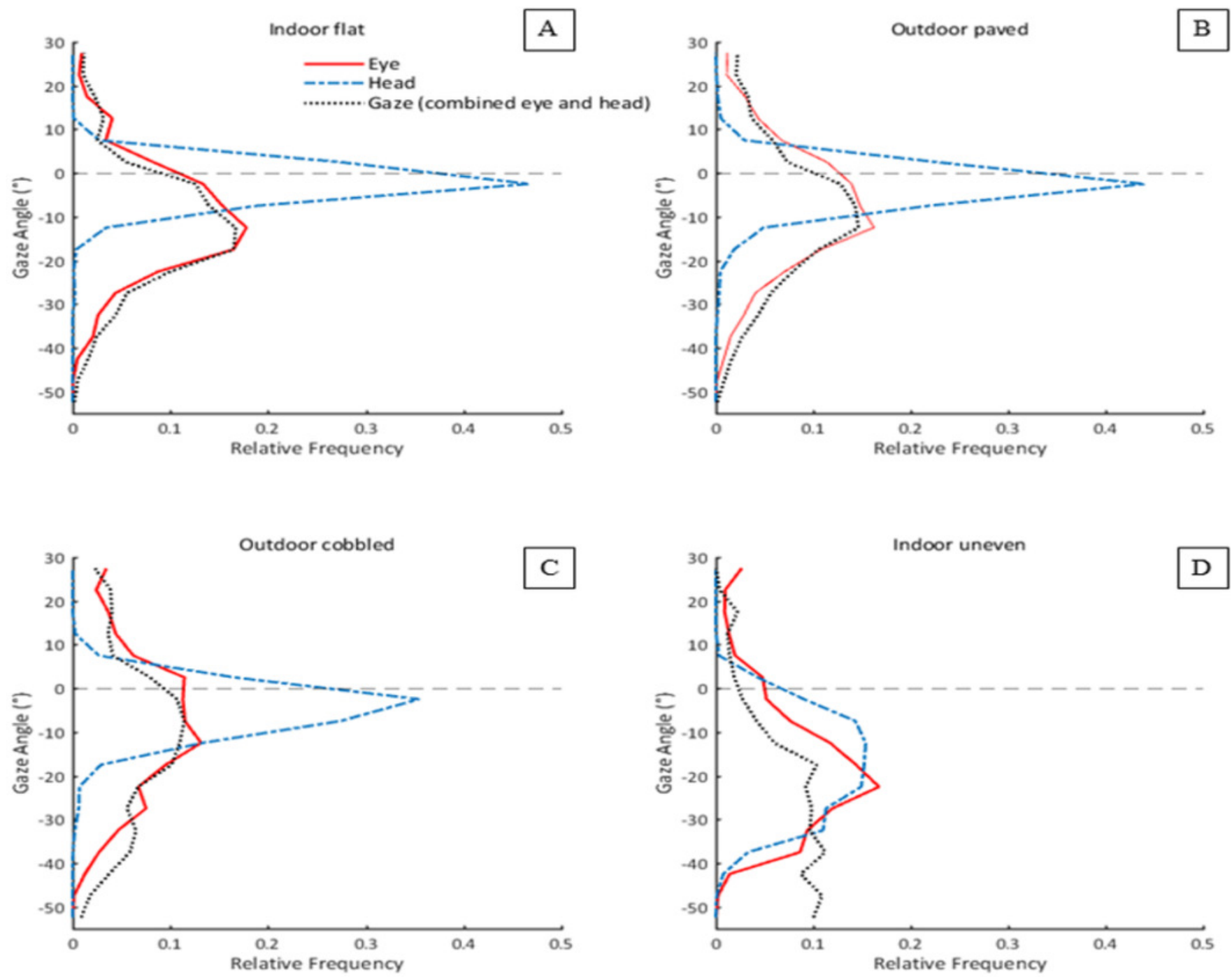
Figure 4

Figure 4

Mean ( \pm SE) gait speed (metres/second) for the four surfaces (indoor, flat, outdoor, paved, outdoor, cobbled and indoor, uneven).

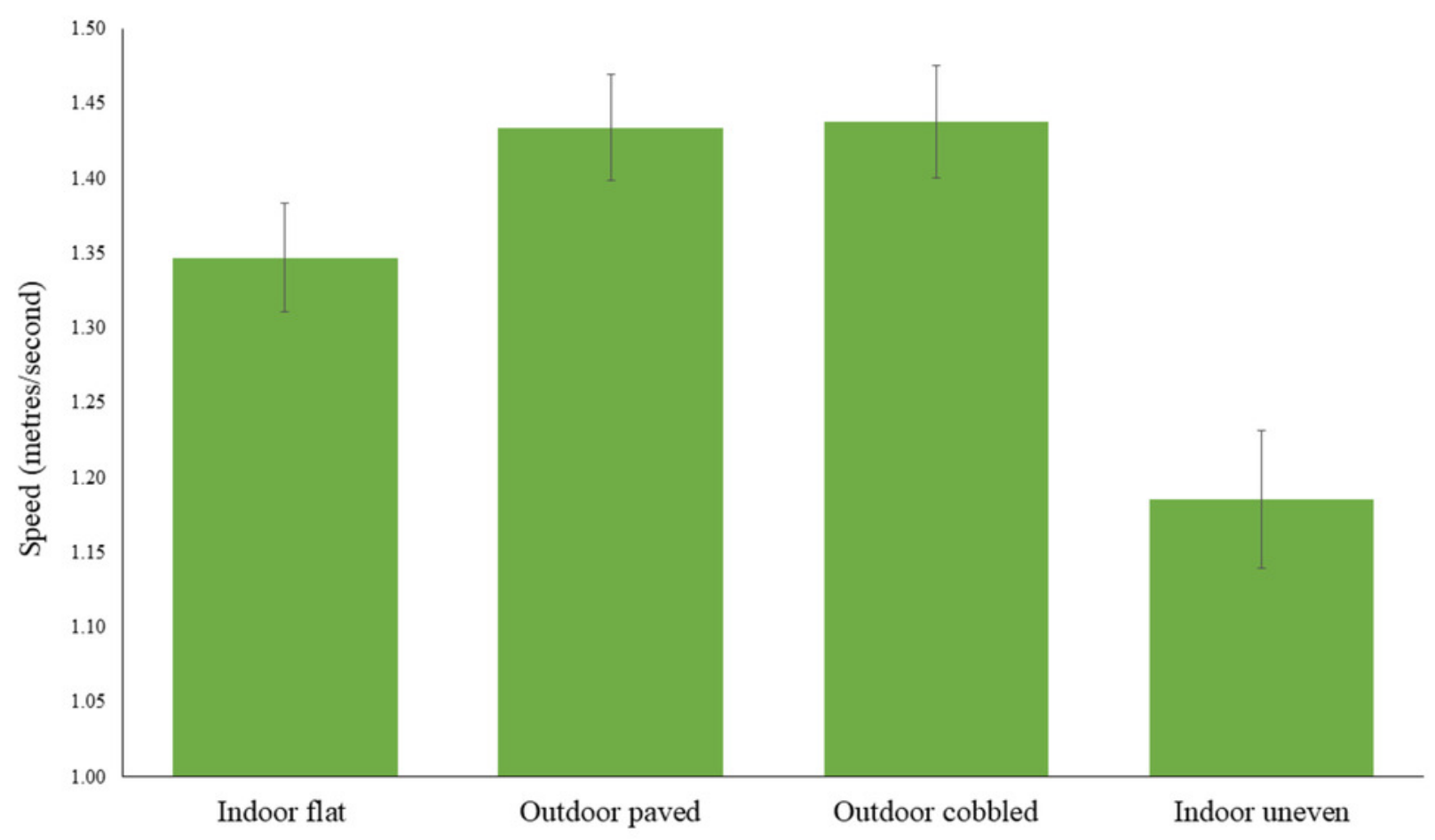




\section{Table 1 (on next page)}

Correlations between mean speed and mean eye, head pitch and gaze angle $\left({ }^{\circ}\right)$. 
1 Table 1: Correlations between mean gait speed and mean eye, head pitch and gaze angles $\left(^{\circ}\right)$.

\begin{tabular}{|c|c|c|c|c|c|}
\hline & & Indoor, flat & $\begin{array}{l}\text { Outdoor, } \\
\text { paved }\end{array}$ & $\begin{array}{l}\text { Outdoor, } \\
\text { cobbled }\end{array}$ & $\begin{array}{l}\text { Indoor, } \\
\text { uneven }\end{array}$ \\
\hline \multirow{3}{*}{$\begin{array}{c}\text { Eye angle } \\
\left(^{\circ}\right)\end{array}$} & $\begin{array}{l}\text { Mean } \\
( \pm \mathrm{SD})\end{array}$ & $+0.01( \pm 0.29)$ & $-0.14( \pm 0.37)$ & $-0.04( \pm 0.39)$ & $+0.09( \pm 0.28)$ \\
\hline & $\mathrm{t}$ value & 0.08 & -1.21 & -0.32 & 1.07 \\
\hline & $p$ value & 0.936 & 0.255 & 0.755 & 0.311 \\
\hline \multirow{3}{*}{$\begin{array}{l}\text { Head pitch } \\
\text { angle }\left(^{\circ}\right)\end{array}$} & $\begin{array}{l}\text { Mean } \\
( \pm \mathrm{SD})\end{array}$ & $-0.08( \pm 0.37)$ & $+0.09( \pm 0.36)$ & $-0.06( \pm 0.41)$ & $-0.11( \pm 0.35)$ \\
\hline & $\mathrm{t}$ value & -0.69 & 0.83 & -0.52 & -1.07 \\
\hline & $p$ value & 0.508 & 0.428 & 0.613 & 0.310 \\
\hline \multirow{3}{*}{$\begin{array}{c}\text { Gaze } \\
\text { angle }\left(^{\circ}\right)\end{array}$} & $\begin{array}{l}\text { Mean } \\
( \pm \mathrm{SD})\end{array}$ & $+0.03( \pm 0.33)$ & $+0.06( \pm 0.35)$ & $0.06( \pm 0.41)$ & $+0.07( \pm 0.32)$ \\
\hline & $\mathrm{t}$ value & 0.28 & 0.54 & 0.46 & 0.67 \\
\hline & $p$ value & 0.789 & 0.601 & 0.654 & 0.516 \\
\hline
\end{tabular}

2 


\section{Table 2 (on next page)}

Mean $( \pm S D$ ) time in seconds and mean number of steps to reach the location that participants were looking ahead to. 
1 Table 2: Mean ( \pm SD) time (seconds) and mean number of steps to reach the location that 2 participants were looking ahead to.

\begin{tabular}{lllll}
\hline & Indoor, flat & Outdoor, paved & Outdoor, cobbled & Indoor, uneven \\
\hline Look ahead time & 6.37 & 7.88 & 6.82 & 2.23 \\
$(\mathrm{sec})( \pm S D)$ & $( \pm 0.12)$ & $( \pm 0.12)$ & $( \pm 0.12)$ & $( \pm 0.15)$ \\
Look ahead step & & & & \\
number & 6.12 & 7.75 & 6.66 & 2.00 \\
$( \pm S D)$ & $( \pm 0.22)$ & $( \pm 0.29)$ & $( \pm 0.25)$ & $( \pm 0.07)$ \\
\hline
\end{tabular}

3 